\title{
Physical Knot Theory: An Introduction to the Study of the Influence of Knotting on the Spatial Characteristics of Polymers
}

\author{
Kenneth C. Millett* \\ Department of Mathematics, University of California \\ Santa Barbara, CA 93106, USA \\ *E-mail:millett@math.ucsb.edu
}

\begin{abstract}
This paper contains selected topics from four lectures given at the Abdus Salam International Centre for Theoretical Physics in May 2009. We introduce the study of the influence of knotting and linking on the spatial characteristics of linear and ring polymer chains with examples of scientific interest. We describe a few basic concepts of the geometry and topology of knots and measures of the spatial shape of open and closed polymer chains. We then present some fundamental mathematical results concerning them. Next we discuss random sampling methods of collections of open and closed chains that are employed to provide estimates of the spatial properties of the chains. Finally, we discuss implementations of the sampling algorithms, survey consequences of theoretical and experimental results, and discuss some interesting problems deserving further research.
\end{abstract}

Keywords: knot, polymer, macromolecule, shape, spatial characteristics, open and closed chains

\section{Introduction}

Humans have long exploited the special properties of polymer chains to manufacture materials used in their everyday life. Polymers are very large molecules existing in a wide variety of topological types, both singly or in collections. While they exhibit substantial structural regularity, e.g. DNA Figure 1, their large scale topology can be linear or circular in nature (the cases of principal interest here) or resemble quite complex graphs, for example star or wheel graphs. They can be natural, e.g. silk, cotton, shellac, rubber, amber, and the shells of crabs and lobsters or synthetic, e.g. bakelite, nylon, neoprene, polystyrene, and polypropylene to name just a few. They are found everywhere, including in the DNA, proteins, and other molecular structures that make up our bodies.

A common mathematical model for polymers is a polygonal chain, i.e 
a collection of vertices and connecting edges. The polygons can be open, i.e. topologically equivalent to a closed interval, or closed, i.e. topologically equivalent to a circle. They are studied individually or in large collections. From the topological perspective, a polygonal ring in 3-space defines a knot while a collection determines a link. The key feature that distinguishes physical knot theory from the classical knot theory is that there are constraints on the chains arising from the physical properties of the polymers being studied. For example, the heterogeneity of the polymer may lead one to study equilateral polygons. The material properties may impose constraints on the bending or twisting of the polygon. The thickness of the material may impose a requirement that the collection of small tubular neighborhoods of the polygons consist of mutually disjoint tori. The imposition of these constraints gives birth to a whole new class of mathematical problems that are not simply of a topological nature but include geometric features in fundamental ways.

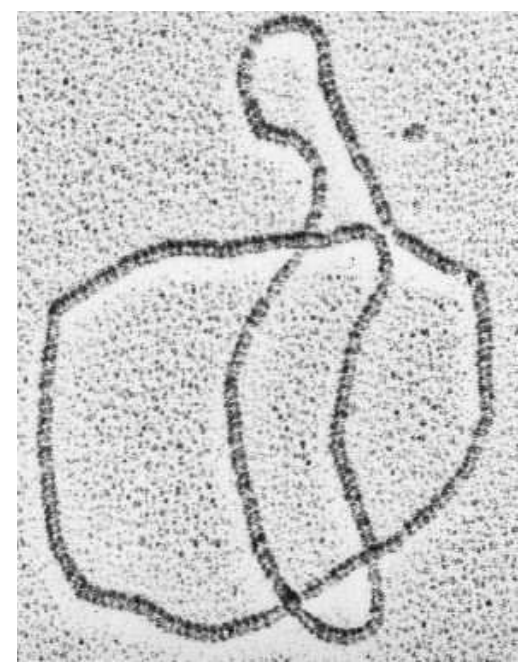

Fig. 1. Electron micrograph of a RecA protein coated DNA trefoil knot generated by E. coli DNA topoisomerase acting on nicked circular DNA. Micrograph courtesy of Andrzej Stasiak, University of Lausanne

What is the relationship between knotting and linking, the average topological structure or geometric structure of configurations, and the biological or physical properties or the functionality of the polymers they model? What sorts of measures does one employ to characterize the shape of poly- 
mers? We will discuss a few of these questions, beginning with those more frequently studied: the identification of the knot type, the radius of gyration, and the average crossing number. The identification of knotting in ring polymers and the extension to knotting in open chains due to applications to protein structures is central. In addition to the specific type of knotting, we will be interested in the average scale of the knotting and the average shape of the knots. Furthermore, we will look at slipknots, i.e. knotted segments of a polymer chain which are contained in a larger unknotted chain, and their properties.

While there are some key results for which we have rigorous proofs, much of the information comes from the random sampling of the space of all possible configurations. As a consequence, we will describe two standard strategies, the polygonal fold method and the crankshaft rotation method, and the rigorous proofs that they properly sample the set of configurations. Implementations of these methods have provided a rich set of data whose analysis provides estimates of the shape parameters discussed earlier. A discussion of the consequences of this information and their implications for future research will complete this introduction to physical knot theory.

\section{Models of biological and physical polymers}

In this section, we will focus attention on structures that are locally linear such as found in models for DNA and proteins, see Figure 1, where we replace the sugar-phosphate backbone or related structures by a linear chain of vertices and edges. We will require that there is no "branching" or "crosslinking" in the structure.

\subsection{Intrinsic structure}

The molecular chains can be modeled as unions of linear segments (polygonal arcs) whose lengths, vertex angles, and torsion statistically reflect the structural properties of the polymer, e.g. Kuhn length. We will assume that the polymers are homogeneous so that, in our polygonal models, the edge lengths will be equal to unity. The individual chains can be either open, i.e. topologically equivalent to closed intervals, or closed, i.e. topologically equivalent to circles. A chain, $K$, is described by its vertex set $\left\{v_{1}, v_{2}, \ldots, v_{n}\right\}$ with edges connecting a vertex with it successor and, if the chain is closed, the last vertex with the first. Thus, if $\left\{v_{1}, v_{2}, v_{3}, \ldots, v_{n}\right\}$ are vertices, the edge vectors of a closed chain $\left\{e_{1}=v_{2}-v_{1}, e_{2}=v_{3}-v_{2}, \ldots, e_{n}=v_{1}-v_{n}\right\}$. Note that an open chain of 
$n$ edges has one more vertex than a closed chain of $n$ edges. Examples of 50 edge open and closed chains with initial vertex at $\{0,0,0\}$ are shown in Figure 2.

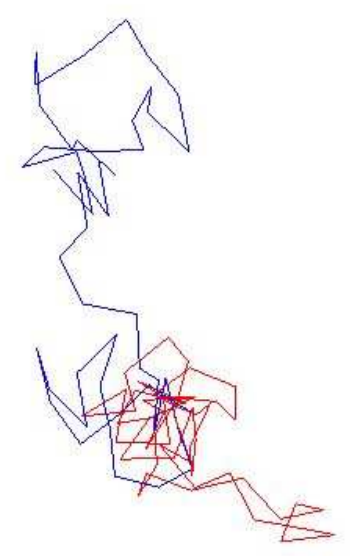

Fig. 2. Linear and circular 50 edge chains

\subsection{Extrinsic structure of closed chains}

The conformations of closed polygonal chains in 3-space can be different in many distinct ways. Indeed, this is also the case for open chains if one focuses on individual "frozen" conformations rather than allowing deformations without self intersections. By allowing for changes in lengths and bending of edges, one arrives at the topological notion of knotting and linking.

A closed (circular) chain is knotted if it can not be deformed, in a structure preserving manner, to the standard planar equilateral polygon of that number of edges, Figure 3. Two closed chains represent the same knot type if one chain can be deformed to the other in a structure preserving manner. If it is the topological structure that is to be respected, one studies the notion of topological knotting. If, however, one must preserve the length of the edges, a geometric stucture has been imposed and the result is the distinctly different concept of geometric knotting. A particularly elusive 
example was the $8_{19}$ eight edge equilateral polygonal knot, Figure 8 , whose existence I proved via numerical methods. Note that, in these figures, care is paid to insure that the 2-dimensional images of the knot accurately capture the structure of the 3-dimensional polygonal chain by insuring that image is unchanged under small changes of perspective and that the under/over crossing structure is clearly discernible, see Figure 3. Such images are called knot presentations.
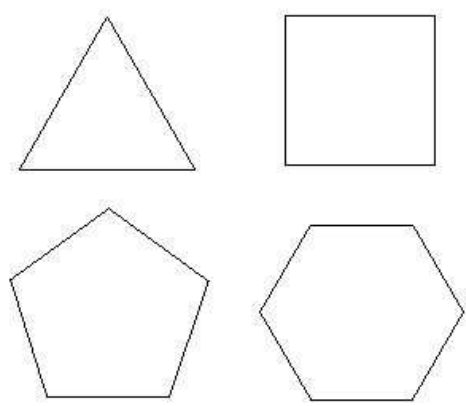

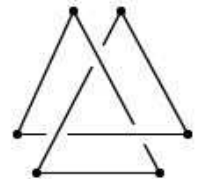

$3_{1}$

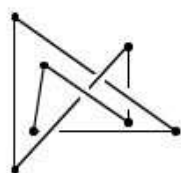

$4_{1}$

Fig. 3. Standard regular polygons, right, and the six edge equilateral trefoil and the seven edge equilateral figure-eight knots

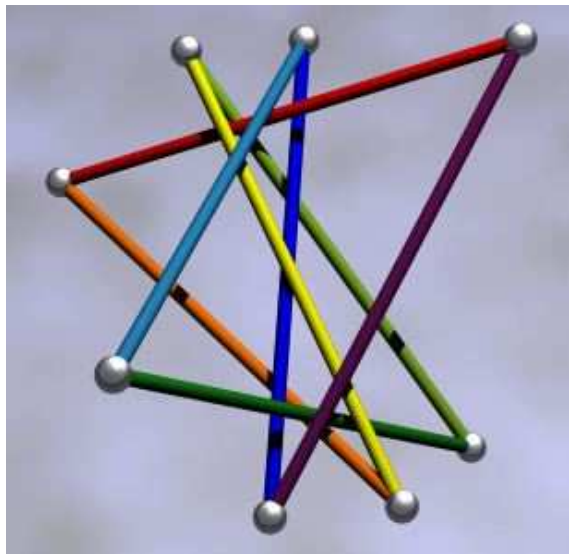

Fig. 4. An octagonal equilateral 819 , Image provided by Peterson Tretheway 


\subsection{Extrinsic structure of open chains}

In our study of the spatial structure of open chains, we emphasize that this concerns the extrinsic properties of the "frozen" configuration. Knotting or linking of open chains is not invariant under topological deformations. For chains in which the edges are of unequal length, there are examples of knotting and linking, ${ }^{1-6}$ under deformations that respect the linear structure and perserve lengths of edges. Whether or not this is true for open equilateral chains is unknown.

Problem 2.1. Are there knotted open equilateral chains?

Thus, our open equilateral chains will be frozen in position unless explicitly stated otherwise. To detect knotting in such open chains, I prefer to consider the statistics of closures of the chain to the "sphere-at-infinity". ${ }^{7-9}$ There are many other strategies that have been employed. ${ }^{10-19}$ These include some which "shorten" the open chain by means of an algorithm that often includes choices that lead to some level of indeterminacy as to the final result. Such uncertainty leads to concerns that must be addressed in applications of these methods. One such example is shown in Figure 5. Here, if one applies a popular shortening method, ${ }^{14}$ the outcome depends upon the end at which one starts. Another strategy is to simply connect the end vertices to a fixed point, directly to each other, or to "infinity". Another looks for solid balls capturing the "knotted" portion in its interior. These methods can lead to an indentification of knotting that is not consistent with what might expect considering the large scale structure of the conformation or are of such limited application that they are difficult to use in these contexts. While it is possible that the extent of indeterminacy that arises in some of these methods is insufficiently large as to undermine the analysis, this is difficult to assess. As a consequence, I prefer the statistically robust method connecting the ends of the chain to points on the "sphere-at-infinity." For all practical purposes, one may consider this to be a sphere of very large radius, compared to the diameter of the conformation, centered at the centroid of the chain, see Figure 5. By applying this method to subchains of closed chains, one can identify the site and scale of knotting in a large chain.

Thus, to identify knotting in open chains, one collects a random sample of closures of appropriate size, computes a knot invariant such as the HOMFLYPT polynomial, ${ }^{20}$ Figure 6 , to identify the topological knot type of each closure, and analyzes the resulting collection of knot types (actually their polynomial surrogates). These statistics give the spectrum of knotting 

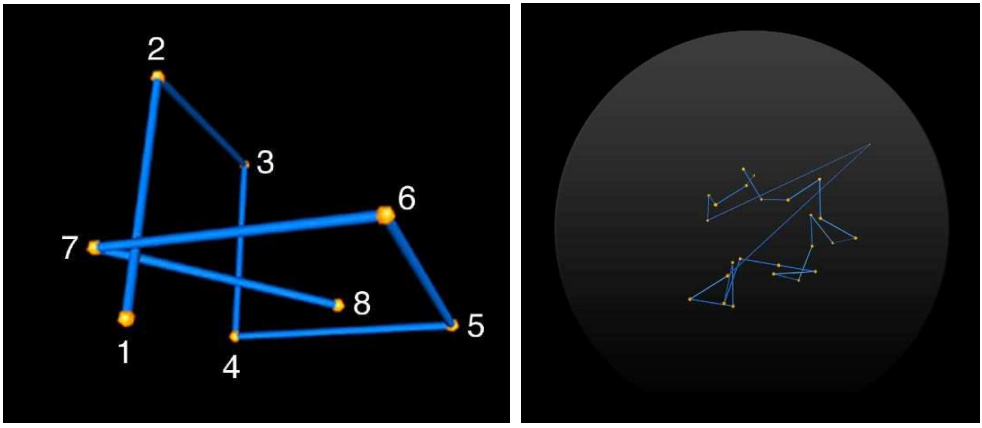

Fig. 5. Curve shortening example and a "sphere-at-infinity" closure example ${ }^{7}$

of the chain. The selection an appropriate threshold to establish the knot type of the open chain, for example 0.6 , results in the identification of a trefoil knot for the spectrum shown in Figure 7.

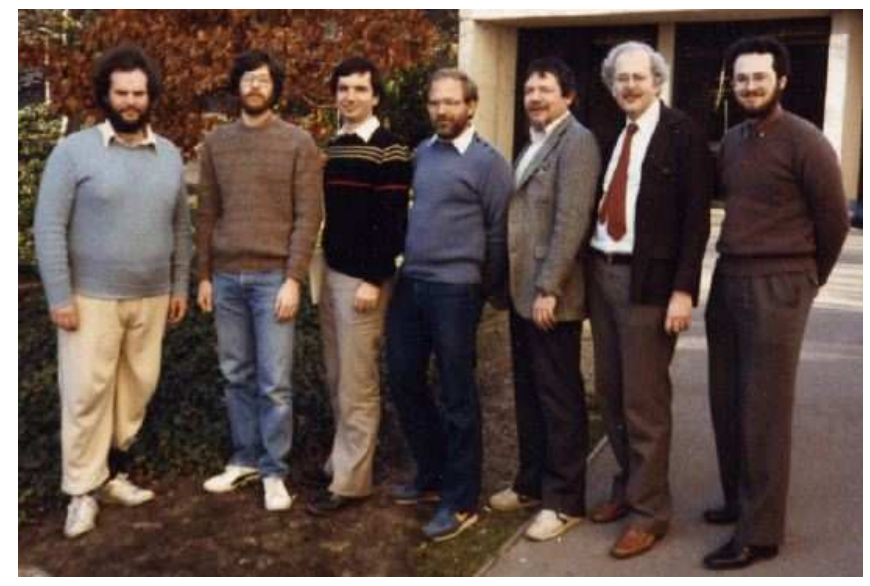

Fig. 6. In Berkeley, summer 1985, Vaughan Jones with James Hoste, Adrian Oceanu, Kenneth Millett, Peter Freyd, W. B. Raymond Lickorish, and David Yetter (HOMFLY) Absent were J. Przytycki and P. Traczyk

\section{Elements of physical knot theory}

In applications of polygonal models, both open and closed chains, to the study of physical or biological polymers, many aspects of the topological 


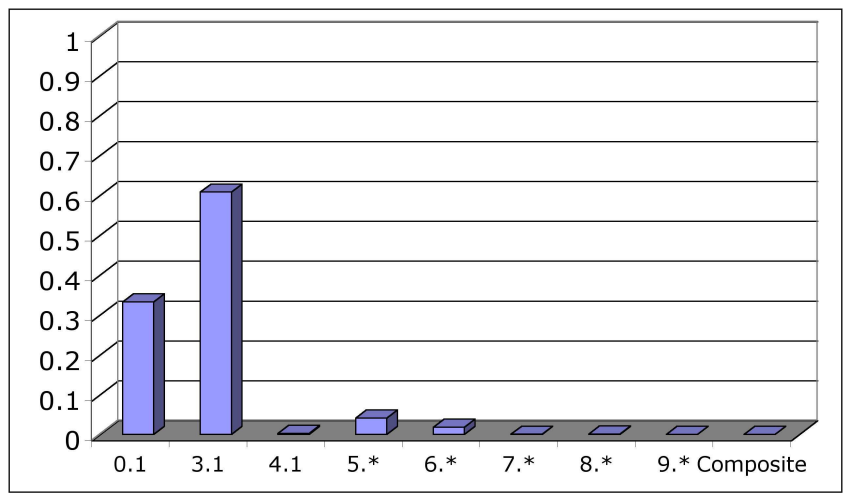

Fig. 7. A sample spectrum of the knotting of an open chain. ${ }^{8}$ The trivial knot and trefoil account for more than $90 \%$ of the sample with the remainder being, principally, five and six crossing knots.

and geometrical structures have been employed. Some of these arose in the topological study of knots while others have developed in response to the goal of modelling physical and biological properties.

\subsection{Average crossing number}

The $A C N(K)$ is understood as the average of the crossing number of a configuration over all orthogonal planar projections. These planes are parametrized by their normal unit vectors, i.e. points on the unit sphere.

$$
A C N(K)=\frac{1}{4 \pi} \int_{S^{2}} \operatorname{cr}(v) d A
$$

If the curve is parameterized by $\gamma(t)$, the $A C N$ is given by the following integral:

$$
\operatorname{ACN}(K)=\frac{1}{2 \pi} \int_{S^{1}} \int_{S^{1}} \frac{\left|\left(\gamma^{\prime}(t), \gamma^{\prime}(s), \gamma(t)-\gamma(s)\right)\right| d t d s}{\|\gamma(t)-\gamma(s)\|^{3}}
$$

in which $\left|\left(\gamma^{\prime}(t), \gamma^{\prime}(s), \gamma(t)-\gamma(s)\right)\right|$ denotes the absolute value of the triple vector product and \|\| denotes the vector norm in $\mathbf{R}^{3}$.

\subsection{Curvature and torsion}

If $\gamma(s)$ is an arc length parameterization of a curve, ${ }^{21,22} T(s)=\frac{d \gamma(s)}{d s}$. The curvature is defined by $\kappa(s)=\left\|\frac{d T(s)}{d s}\right\|$. The nomal vector is $N(s)=\frac{\frac{d T(s)}{d s}}{\left\|\frac{d T(s)}{d s}\right\|}$. 


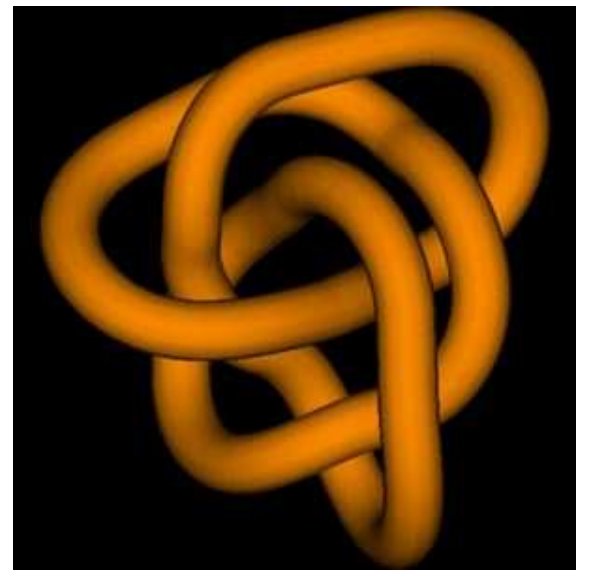

Fig. 8. An 819 knot with ACN 11.2271

The binormal vector is $B(s)=T(s) \times N(s)$. The torsion is defined by $\tau(s)=-\left\|\frac{d B(s)}{d s}\right\|$.

\subsection{Total curvature and total torsion}

For spatial curves we define the total curvature and the total torsion to be the integrals of the curvature and torsion as follows:

$$
\begin{aligned}
& \operatorname{TCU}(K)=\int_{S^{1}} \kappa(s) d s \\
& T T O(K)=\int_{S^{1}} \tau(s) d s
\end{aligned}
$$

A closed curve must have total curvature at least $2 \pi$. The total curvature and total torsion of the knot in Figure 8 are 26.4085 and 118.602, respectively.

Theorem 3.1 (Fary-Milnor ${ }^{23}$ ). If $K$ is a smooth non-trivial knot, its total curvature is greater than $4 \pi$.

As our models are based on polygonal chains, we translate these concepts to the polygonal setting. The $\left\{v_{1}, v_{2}, \ldots, v_{n+1}\right\}$ are the $n+1$ vertices of an $n$ edge polygonal chain. These give the edge vectors $\left\{e_{1}=v_{2}-v_{1}, e_{2}=\right.$ $\left.v_{2}-v_{2},, e_{n}=v_{n+1}-v_{n}\right\}$ for an open chain. If the chain is closed, we set $v_{1}$ 
equal to $v_{n+1}$. The angle, in radians, between $e_{i}$ and $e_{i+1}$ defines the local curvature at $v_{i+1}$. The total curvature is defined to be the sum of these angles (measured in radians). Adjacent vectors, $e_{i}$ and $e_{i+1}$, determine a plane. The angle between adjacent planes defines the torsion at each edge. The total torsion is the sum of these angles.

\subsection{Radius of gyration}

The center of mass and squared radius of gyration of a chain, $K$ are defined by:

$$
\begin{gathered}
C M(K)=\frac{\Sigma v_{i}}{n} \\
R G N(K)^{2}=\frac{\Sigma\left\|v_{i}-C M(K)\right\|^{2}}{n}
\end{gathered}
$$

The $R G N$ is one of the most frequently employed measures of the spatial extent of a configuration. The radius of gyration of the $8_{19}$ knot in Figure 8 is 3.165 .

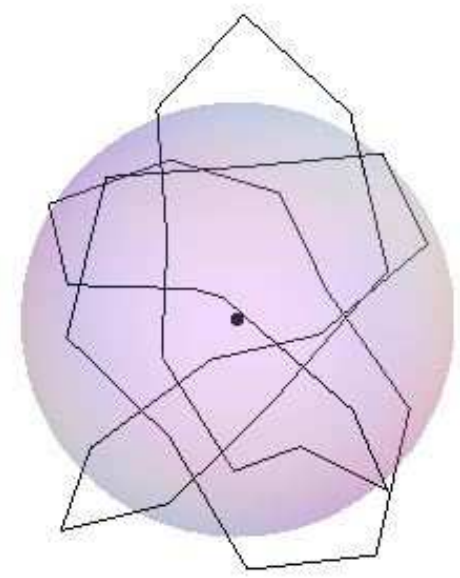

Fig. 9. The center of mass and the radius of gyration sphere associated to a polygonal trefoil 


\subsection{Injectivity radius of a knot in 3-space}

The injectivity radius of a smooth knot is 3-space is the upperbound of the radii of embedded normal tubes centered on the knot. ${ }^{24}$ This radius is determined by limits imposed by the curvature and by contact points of distance portions of the normal tube 11. Gonzalez and Maddocks ${ }^{25}$ give a very general definition as follows: For any triple of distinct points in 3space, let $r(x, y, z)$ be the radius of the unique circle they determine. If $T(x)$ is a line tangent to the curve at $x$, let $r(T(x), y)$ be the radius of the unique circle tangent to $T(x)$ at $x$ and containing $y . \rho_{x}(L)$ is the infimum of $r(x, y, z)$ over distinct points $x, y, z$ with $x$ fixed. For a smooth link, the function $\rho_{x}(L)$ is continuous and $0<\rho_{x}(L)<\frac{1}{\kappa(s(x))}=\rho(s(x))$, where $s(x)$ is the arc length parameter of the point $x$ and $\rho(s(x))$ is defined to be the radius of curvature of $L$ at $x$. The thickness, $\rho(L)$, is the infimum of $\rho_{x}(L)$ over all $x \epsilon L$.

The images of thick knots often do not display the maximal tubes due to the difficulty of distinguishing features of the structure, see Figure 10.
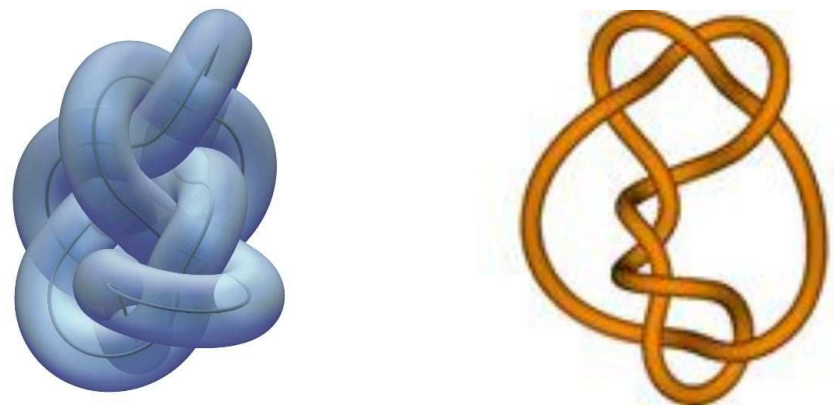

Fig. 10. Two images of thick 86 knots. Images provided, respectively, by Cantarella and Scharein

For polygonal knots, Eric Rawdon, ${ }^{26-28}$ has defined a polygonal injectivity radius reminiscent of the smooth category in terms of the vertex angles and the minimal distal distances.

\section{Theoretical results on the random sampling of equilateral open and closed chains}

Due to biological, physical, geometric, and topological interest, the collections of open or closed (or ring) equilateral polygons have attracted much 


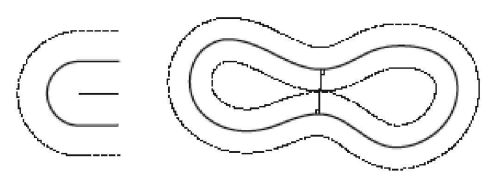

Fig. 11. Injectivity radius constraints ${ }^{26}$

attention. The results of Randell, ${ }^{29,30}$ Calvo, ${ }^{31-35}$ among others, tell us exactly which knots can be represented by closed chains with eight or fewer edges. Beyond eight edges, however, there are only estimates for the number of knots representable with exactly $n$ edges. The frequency of a specific knot type, for a fixed number of edges, is another quantity that has not been determined rigorously. It has only been estimated via numerical studies. Generating and analyzing large random samples is, therefore, a fundamental component of the modeling of macromolecules. The theoretical results presented here should be considered as a first step toward models that take curvature, torsion, and injective radius into consideration.

The space of open embedded equilateral chains is the same as the space of embedded random walks. In the following, we will first consider open chains and then those which are closed, i.e. in which the final vertex equals the first vertex.

\subsection{Open chains (or random walks)}

An open chain, based at the origin, is determined by a set of $n$ vertices, $v_{0}=$ $\mathbf{0}, v_{1}, \ldots, v_{n}$. The associated edge vectors are determined by $\mathbf{e}_{i}=v_{i}-v_{i-1}$ and, in turn, determine the vertices. The edge vectors have unit length and, thereby, correspond to points on the unit radius 2-dimensional sphere.

$$
\begin{aligned}
v_{0} & =\mathbf{0}, \\
v_{1} & =\mathbf{e}_{1}, \\
v_{2} & =\mathbf{e}_{1}+\mathbf{e}_{2}, \\
& \vdots \\
v_{n} & =\mathbf{e}_{1}+\mathbf{e}_{2}+\mathbf{e}_{3}+\ldots+\mathbf{e}_{n} .
\end{aligned}
$$

Thus, an $n$-edge equilateral chain can be considered as a point in the product of $n$ 2-dimensional spheres, i.e. 


$$
\left(\mathbf{e}_{1}, \mathbf{e}_{2}, \ldots, \mathbf{e}_{n}\right) \in \mathbf{S}^{2} \times \mathbf{S}^{2} \times \ldots \times \mathbf{S}^{2}
$$

The requirement that the edges meet only at common vertices imposes a collection of constraints that insures that embedded chains define an open subset, $\mathbf{C}_{n}$, of $\mathbf{S}^{2} \times \mathbf{S}^{2} \times \ldots \times \mathbf{S}^{2}$. An example of such a chain is shown in Figure 12. The meaning of "random" is determined by the natural measure on the space of configurations. We will use the product measure on $\mathbf{S}^{2} \times \mathbf{S}^{2} \times \ldots \times \mathbf{S}^{2}$. A random edge vector $\mathbf{e}$ is determined as follows:

\section{Random Chain Generation Method}

(1) With respect to the uniform measure on $[0,1]$, select two numbers, $a$ and $b$.

(2) Define $\alpha=2 \pi a$ and $\beta=\operatorname{ArcCos}[2 b-1]$

(3) Define $\mathbf{e}=(\operatorname{Cos}[\alpha] \operatorname{Sin}[\beta], \operatorname{Sin}[\alpha] \operatorname{Sin}[\beta], \operatorname{Cos}[\beta])$.

(4) Check that the addition of e does not introduce any intersections in the chain defined to that point. If so, reject the vector and try again.

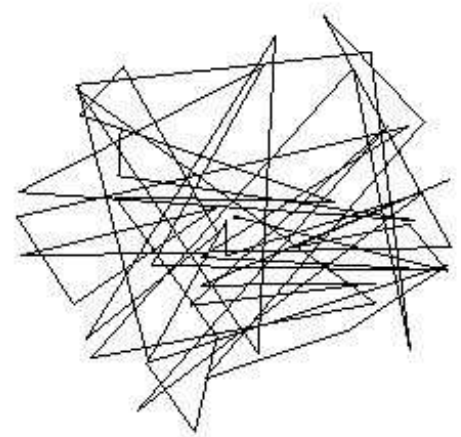

Fig. 12. A "random" 50 edge chain in 3-space

\subsection{Closed chains (or polygons) in 3-space}

Closed chains are determined by a list of unit vectors $\mathbf{e}_{i},\left(\mathbf{e}_{1}, \mathbf{e}_{2}, \ldots, \mathbf{e}_{n}\right) \in$ $\mathbf{S}^{2} \times \mathbf{S}^{2} \times \ldots \times \mathbf{S}^{2}$. The requirement that $v_{n}=\mathbf{0}$ is equivalent to $\mathbf{e}_{1}+\mathbf{e}_{2}+\mathbf{e}_{3}+\ldots+\mathbf{e}_{n}=\mathbf{0}=(0,0,0)$. This imposes three relations on the set of edges thereby decreasing the dimension to $2 n-3$. The requirement that the edges meet only at common vertices imposes a collection of constraints 
that insures that the set of embedded closed chains defines an open subset, $\mathbf{P}_{n}$, of the $2 n-3$ dimensional subset of $\mathbf{S}^{2} \times \mathbf{S}^{2} \times \ldots \times \mathbf{S}^{2}$. An example of a "random" twenty-edge equilateral polygon is show in Figure 13.

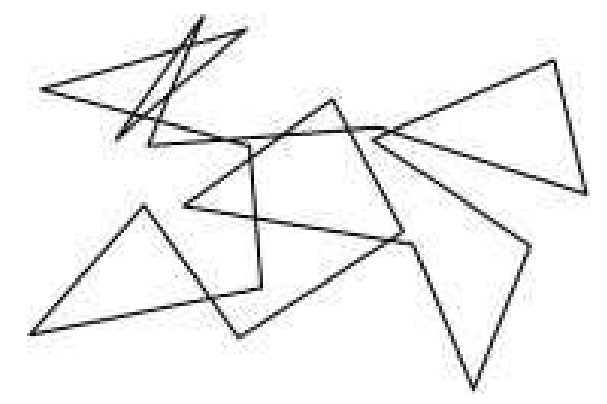

Fig. 13. A "random" 20 edge equilateral polygon in 3-space

\subsection{An important property of these spaces}

These spaces can be considered as subsets of the product of $n$ copies of $\mathbf{R}^{3}$. As sampling occurs via numerical procedures which are not exact, small numerical "errors" naturally arise. Such "errors" represent "approximations" of the coordinates of the vertices of the polygonal chain. They can also be considered as corresponding to perturbations of exact data. These errors can impact the conclusions that one may wish to infer from an analysis of the data. Thus, a fundamental question that one must ask is "How close to having an equilateral polygon with specified properties must one be in order to insure the mathematical existence of a nearby equilateral polygon with exactly these topological or geometric properties?" Suppose that $P$ is an $n$ vertex polygon, $v_{0}, v_{1}, \ldots, v_{n}$ and $P^{\prime}$ is another with vertices $v_{0}^{\prime}, v_{1}^{\prime}, \ldots ., v_{n}^{\prime}$. We measure the distance between $P$ and $P^{\prime}$ by $d\left(P, P^{\prime}\right)=\max \left\{\left\|v_{i}-v_{i}^{\prime}\right\|\right\}$, This metric on $\mathbf{R}^{3 n}$ gives a measure of how close two polygons are. Similarly $\max \left\{\left|\left\|e_{i}\right\|-1\right|\right\}$ is a measure of how close $P$ is to being an equilateral polygon with unit length edges. Answering our question requires the conjunction of several considerations that we shall discuss next.

\subsubsection{Chains and tubes}

We look closely at the injective radius, see Sec. 3.5, to develop a reply for polygonal models. ${ }^{27}$ Let $\left\{\mathbf{v}_{1}, \mathbf{v}_{2}, \ldots, \mathbf{v}_{n}\right\}$ be the vertices of a 3 -space 
polygon, $P$. Let $\|\mathbf{e}\|$ denote the norm of the edge vector $\mathbf{e}$. Given a vertex $\mathbf{v}_{j}$, let angle $\left(\mathbf{v}_{j}\right)$ be the turning angle from prior edge, $\mathbf{e}_{j-1}$, to the next edge, $\mathbf{e}_{j}$. Define

$$
\operatorname{Rad}\left(\mathbf{v}_{j}\right)=\frac{\min \left\{\left\|\mathbf{e}_{j-1}\right\|,\left\|\mathbf{e}_{j}\right\|\right\}}{2 \operatorname{Tan}\left(\frac{\operatorname{angle}\left(\mathbf{v}_{j}\right)}{2}\right)}
$$

and

$$
\operatorname{MinRad}(P)=\min \left\{\operatorname{Rad}\left(\mathbf{v}_{j}\right)\right\} .
$$

For $x \in P$, let $\mathbf{d}_{x}: P \rightarrow \mathbf{R}$ be defined by $\mathbf{d}_{x}(y)=\|x-y\|$. A point, $y$, is a turning point if $\mathbf{d}_{x}$ changes from increasing to decreasing or vice-versa at $y$. Let $D C \in P \times P$ denote the collection of points, $(x, y)$ with $x \neq y$, that are turning points of $\mathbf{d}_{y}$ and $\mathbf{d}_{x}$, respectively. Let

$$
\operatorname{dscd}(P)=\min \{\|x-y\|:(x, y) \in D C\} .
$$

Rawdon ${ }^{26}$ defines the polygonal injective radius as

$$
R(P)=\min \left\{\operatorname{MinRad}(P), \frac{d s c d(P)}{2}\right\} .
$$

The following theorem provides a way to analyze the effect of pertrubations or numerical approximations on the structure of a closed equilateral polygonal chain. The analogous result for open chains easily follows by the same methods.

Theorem 4.1 (The Closed Chain Tube Theorem ${ }^{27}$ ). If $r<R(P)$, the union of solid balls of radius $r$ centered at points on the closed polygon $P$ is an embedded solid torus.

Theorem 4.2 (The Open Chain Tube Theorem). If $r<R(C)$, the union of solid balls of radius $r$ centered at points on the open polygonal chain $C$ is an embedded ball.

These theorems help provide one step in answering our question. 
Theorem 4.3 (The Perturbation Theorem ${ }^{27}$ ). If $P$ is equilateral polygonal chain (open or closed) of constant edge length, $E$, and the scale of a perturbation, $r=d\left(P, P^{\prime}\right)$, is smaller than minimum of the polygonal injective radius of $R(P)$ and $\frac{12 E R(P)^{2}-E^{3}}{8 R(P)^{2}+2 E^{2}}$, then all perturbed polygonal chains, $P^{\prime}$, are geometrically equivalent $P$.

One application of the theorem in response to our questions concerns the existence of an equilateral eight edge 819 knot. Random searches or perturbations of examples, without requiring equal length edges, lead to the impression that this might be an example of a knot whose polygonal stick number was different from its equilateral stick number, i.e. it could be represented as an eight edge polygon but not an equilateral one. An early example was discovered to fail to satisfy the conditions of the Perturbation Theorem, i.e. the edges lengths were not quite equal and, simultaneously, the edges almost crossed so that its injective radius was to small to insure that it could be approximated by an equilateral polygon of the same topological knot type. Later, in an unrelated effort, I developed a new random search strategy to test the existence of this knot. It appeared to be successful and, this time, the Perturbation Theorem confirmed the existence of the configuration shown in Figure 4, whose coordinates are (approximately) the following:

$$
\begin{aligned}
& v_{1}=(0.0,0.0,0.0) \\
& v_{2}=(4.244988698233,-5.29806224161222,4.23209255993696) \\
& v_{3}=(1.621389440625,-2.446558361029,-2.76688254749867) \\
& v_{4}=(6.9553834171687,-3.1449123567692,3.1543343844196) \\
& v_{5}=(-0.58394895866445,-5.81008379802231,2.919114241909) \\
& v_{6}=(3.9278286450686,-2.446558361029,-2.76688254749867) \\
& v_{7}=(2.0591187707439,-5.1915142515365,4.5113823944207) \\
& v_{8}=(5.3795054883023,-5.2350054125733,-2.76688254749867)
\end{aligned}
$$

\subsection{Markov Chain Monte Carlo (MCMC) sampling of the space of closed equilateral chains}

A discrete stochastic process (or random process) is a sequence of random states, $K_{i}$. Markov Chain Monte Carlo sampling refers to discrete methods of sampling a probability distribution, a sample space with a specific unit measure, using a discrete stochastic process whose equilibrium distribution gives the measure on the space. This means that, given any measurable 
subset, the limiting ratio of the number of samples in the subset and the total number of samples approaches the proportional measure of the subset as the number of samples goes to infinity. A Markov chain is a stochastic process for which future samples depend only on the present case or, perhaps, a finite number of previous states within a time interval. The fundamental property of MCMC sampling is that, independent of the initial state, this ratio converges to the measure. A key problem is to determine how many samples are required in order to estimate the desired measure within a predetermined error bound. A good MCMC will require a smaller number of samples, independent of the initial state. Balanced against this is the time required to achieve statistical independence of sample choices. Thus we have The Random Sampling Objective: Provide a $M C M C$ algorithm whose sampling of $P_{n}$ converges to the measure rapidly and with a high degree of accuracy. We will describe several of the most commonly used algorithms for sampling the space of closed equilateral chains.

\section{Algorithm 4.1 (The Basic Algorithm).}

(i) Select an initial configuration, e.g. the standard planar regular polygon.

(ii) Perform a sequence of successive random modification steps giving closed equilateral chains until the sampling criteria are met. Add the configuration to the sampling sequence.

(iii) Replace the configuration with this one and return to the previous step until the desired number of samples is achieved.

Variation: An initial configuration is randomly generated to determine the sample.

The two principal methods differ in the specific choice of the modification step:

\section{Algorithm 4.2 (The Polygonal Fold Modification (PFM) Step).}

(i) Randomly select a pair of non-adjacent vertices of the polygon and select one of the segments they bound.

(ii) Using these vertices to define an axis, randomly rotate the selected segment to define a new polygon.

(iii) Verify that the polygon is embedded to complete the polygonal fold construction.

\section{Algorithm 4.3 (The Crankshaft Rotation Modification (CRM) Step).}

(i) Randomly select a pair of edge vectors, $\mathbf{e}_{i}$ and $\mathbf{e}_{j}$. 
(ii) The random rotation of $\mathbf{e}_{i}$ about axis defined by $\mathbf{0}$ and $\mathbf{e}_{i}+\mathbf{e}_{j}$ defines a new pair of edge vectors, $\mathbf{e}_{i}{ }_{i}$ and $\mathbf{e}_{j}{ }_{j}$, that replace $\mathbf{e}_{i}$ and $\mathbf{e}_{j}$, respectively, to define a new polygon.

(iii) Verify that the polygon is embedded to complete the crankshaft rotation step.

\section{Fundamental Properties.}

- The PFM and CRM steps preserve the lengths of the edges.

- The inverse of a PFM, resp CRM, step is a PFM, resp CRM, step.

- The PFM and CRM algorithms are aperiodic, i.e. the probability that a configuration occurs a second time is zero.

- The PFM and CRM algorithms are positive recurrent, i.e. given any two configuration, there is a finite number of CRM (or PFM) modifications taking the initial configuration to the second. This implies that, given any neighborhood of the second, there is a positive probability of starting at the first and ending in the neighborhood in a finite number of steps.
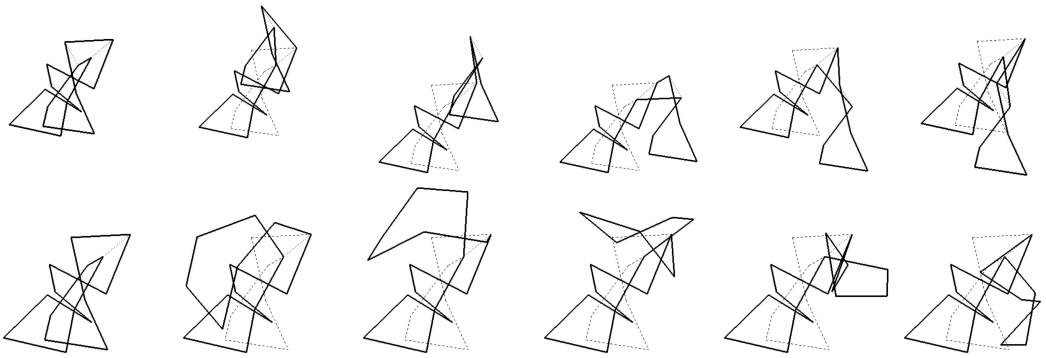

Fig. 14. CRM moves (above) versus PFM moves (below) demonstrating their fundamentally different nature

Figure 14 compares the effects of a crankshaft rotation with those of the polygonal fold. In each vertical pair of frames of this figure, the dashed lines indicate the original position of the polygon. In addition, a pair of dotted lines is drawn joining the endpoints of the two edges selected for the rotation. An animated version of this figure can be viewed at www.avemaria.edu/calvopubs. The modifications of a polygon produced by each method are quite distinct geometric operations. The PFM requires, on average, $\frac{n}{4}$ times as many calculations as CRM. ${ }^{36}$ Each of these methods will asymptotically produce the required sample of the space of equilateral 
polygons as a consequence the following two theorems.

Theorem 4.4. (Millett, ${ }^{37}$ Kapovic $\&$ Milson, ${ }^{38}$ Toussaint ${ }^{39}$ ) Every configuration can be reached from every other configuration by a finite sequence of PFM steps.

Theorem 4.5. (Alvarado, Calvo \& Millett $^{36}$ ) Every configuration can be reached from every other configuration by a finite sequence of CRM steps.

\section{Applications of sampling spaces of open and closed equilateral chains}

We next focus on the application of these random sampling strategies to the acquisition of data illuminating the questions and quantities described earlier, in Section 3. All the methods we have discussed have been employed during one or more of these studies. In particular, the regular polygon, the hedgehog and random triangles, see Figure 15, have been used as starting configurations.

Problem 5.1. How many randomization steps are required before sampling?

We observe that, on average, the PFM requires more calculations per step than the CRM. In our implementation, both PFM and CRM use current sample as the initial polygon for each step and, as a consequence, require some number of modifications before sampling again.
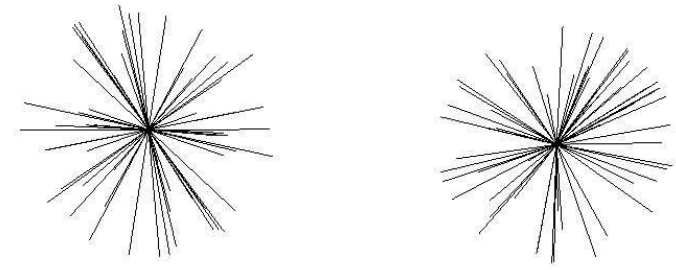

Fig. 15. Initial hedgehog (left) and triangle (right) sets of 60 unit vectors. Although appearing quite similar, the antipodal symmetry of the initial hedgehog data distinguishes the left from the right sets of unit vectors. 


\subsection{Measures of randomness}

One measure of the extent to which an algorithm is random is given by using the maximum Pearson Correlation Index, PCI, to analyze the collection of edge vectors that is generated. We can, thereby, estimate the number of steps needed to achieve a PCI close to that of a completely random process. The PCI (also known as the Pearson Product-Moment Correlation) provides a measure of the degree to which two sets of data are linearly related. ${ }^{40}$ It is defined as the covariance between the two sets of data divided by their standard deviations and, therefore, takes on values in the closed interval $[-1,1]$. PCI values near \pm 1 indicate a strong linear relationship between the data sets, while values near 0 indicate a weak relationship. In our case, we determine the correlation between two $n$-sided polygons by comparing the sequences of $3 n$ coordinates obtained from the $n$ edge vectors. We calculate the PCI for these sequences $n$ times, once for each choice of starting vertex, taking as our final result the maximum absolute value observed. We use the absolute value of the Pearson Correlation in order to estimate its average value over a large populations of pairs of configurations. As the standard of comparison, we report the average value for a collection of random vectors equalling the number of edges in the polygons.

Table 1. Edge vector correlations (PCI) for the Crankshaft Rotation Method using various sampling rates. ${ }^{36}$

\begin{tabular}{|c||c|c|c|c|c||c|c|}
\hline \multicolumn{1}{|c||}{ edges } & \multicolumn{6}{c||}{ crankshaft rotations } & \multicolumn{2}{c|}{ random } \\
\hline$n=12 k$ & $n$ & $2 n$ & $3 n$ & $4 n$ & $5 n$ & mean & std dev \\
\hline \hline 12 & 0.3457 & 0.3371 & 0.3354 & 0.3377 & $\mathbf{0 . 3 3 3 0}$ & 0.3280 & 0.0828 \\
\hline 24 & 0.2737 & 0.2665 & 0.2648 & 0.2673 & $\mathbf{0 . 2 6 7 9}$ & 0.2695 & 0.0605 \\
\hline 36 & 0.2326 & $\mathbf{0 . 2 3 4 6}$ & 0.2339 & 0.2307 & 0.2302 & 0.2352 & 0.0484 \\
\hline 48 & $\mathbf{0 . 2 1 0 6}$ & 0.2086 & $\mathbf{0 . 2 1 0 6}$ & 0.2089 & 0.2093 & 0.2127 & 0.0413 \\
\hline 60 & $\mathbf{0 . 1 9 4 6}$ & 0.1931 & 0.1919 & 0.1932 & 0.1928 & 0.1972 & 0.0360 \\
\hline 72 & $\mathbf{0 . 1 8 1 1}$ & 0.1808 & 0.1786 & 0.1797 & 0.1808 & 0.1836 & 0.0319 \\
\hline 84 & 0.1696 & 0.1692 & 0.1692 & $\mathbf{0 . 1 6 9 9}$ & 0.1694 & 0.1732 & 0.0289 \\
\hline 96 & 0.1623 & 0.1614 & $\mathbf{0 . 1 6 2 8}$ & 0.1609 & 0.1612 & 0.1650 & 0.0269 \\
\hline 108 & 0.1546 & 0.1542 & $\mathbf{0 . 1 5 5 4}$ & 0.1535 & 0.1550 & 0.1574 & 0.0247 \\
\hline 120 & $\mathbf{0 . 1 4 9 8}$ & 0.1459 & 0.1480 & 0.1490 & 0.1497 & 0.1510 & 0.0236 \\
\hline
\end{tabular}

Alvarado et. al. ${ }^{36}$ have shown that a conservative sampling rate of $4 n$ crankshaft rotationsis adequate for polygons through 120 edges. In fact, a sampling rate of $n$ crankshaft rotations is sufficient to achieve PCI values within one standard deviation of the random distribution without the closure condition. 


\subsection{Estimation of knot populations}

One of the more challenging problems of statistical knot theory is the estimation of the population of knots that can be realized as an equilateral $n$-gon. As important, for physical knot theory, is the determination of the probability distribution function, $P D F$, of the knot types that are realized for a fixed number of edges and, for a fixed knot type, as a function of the number of edges. To estimate the $P D F$, we first use one of the random knot generation methods. Next we employ topological invariants which are sufficiently effective discriminators of knots and that can be calculated sufficiently easily within the target population. With Bruce Ewing, I developed a computer code in 1985 to calculate the HOMFLYPT polynomial, ${ }^{20,41-44}$ see Figure 6 . This continues to be a very effective tool. A very clear picture has emerged from an analysis of the resulting data, see Figure 16.

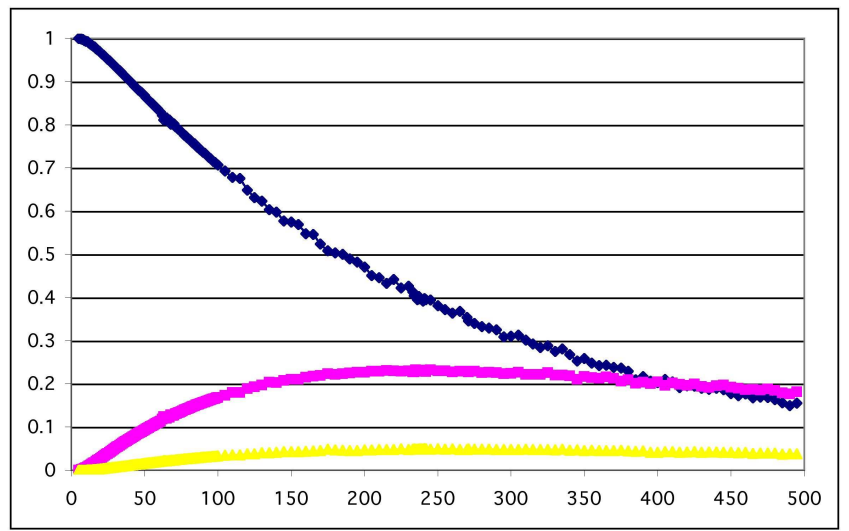

Fig. 16. The probability of a knot type (distinct HOMFLYPT polynomials) as a function of the number of edges: $0_{1}, 3_{1}$, and $4_{1}$. Sample size: $2.51 \times 10^{9}$

Problem 5.2. As a function of the number of edges, how many distinct knot types are represented by equilateral polygons of length $n$ ?

The estimation of the number of distinct knot types and their relative probability is also difficult. ${ }^{45,46}$ Although we know that there are only finitely many knot types, there is no useful estimate of the number of topological knot types as a function of the number of edges. Analysis of knot populations, see Figure 17, shows that even with a relatively large sample 
size, here $2.51 \times 10^{9}$, there are many knot types (or HOMFLYPT polynomials) that are observed only once. Despite knowing that this number is bounded, an analysis of the number of knots as a function of the sample size, shown in Figure 18, does not yet suggest asymptotic convergence to a finite population much less provide the means to estimate the size of the population.

Problem 5.3. What is the probability distribution of the knot types occuring in equilateral $n$-gons?

Another objective is the estimation of the probability of a fixed knot type as a function of the number of edges. One can prove that the probability of each fixed knot type goes to zero exponentially as the number of edges goes to infinity. There are numerical estimates of the exponential constant for some models and for some of the more likely knot types, e.g. $0_{1}, 3_{1}$, and $4_{1}$. Although one might conjecture that there is an elementary functional model for the probability distribution, Figure 16, this is apparently not the case due to differences in behaviour between the finite range and the asymptotic range. ${ }^{47}$

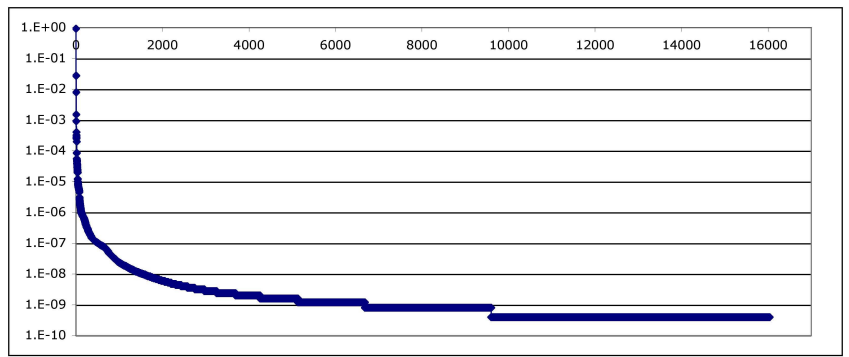

Fig. 17. The observed probability distribution function for distinct HOMFLYPT polynomials of polygons with 32 edges. Total knots 16,497 Sample size $2.51 \times 10^{9}$

\subsubsection{The probability of knotting in 3-space random walks.}

We may also use the HOMFLYPT invariant, as indicated earlier, study knots and slipknots in random walks through the knot spectrum. Just as in the case of random polygons, we know that the probability that random walk contains a knot (slipknot) goes to one as its length goes to infinity. $^{9,10,48,49}$ 


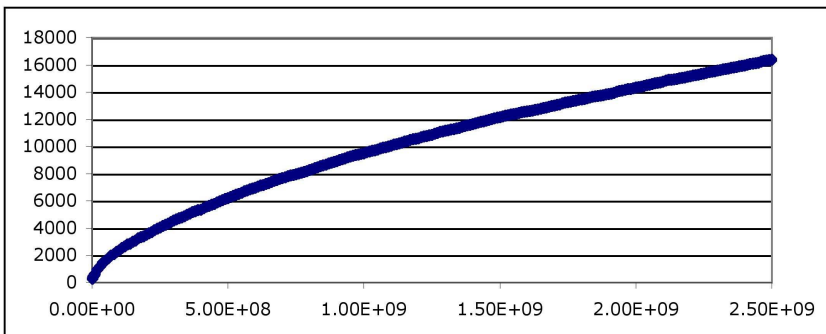

Fig. 18. The number of observed knots as a function of the sample size. Total knots 16,457 Sample size $2.51 \times 10^{9}$

Problem 5.4. How does knotting in random walks compare to that in random polygons?

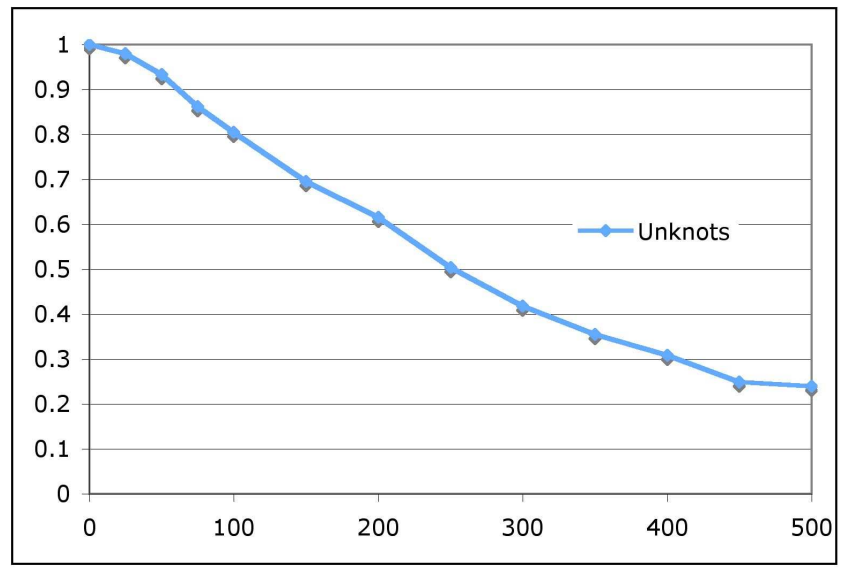

Fig. 19. Knotting in random walks ${ }^{7,8}$

\subsection{Knot and slipknot localization}

Problem 5.5. What is the average size of the knotted (or slipknotted) segments of a random chain or polygon?

One can also employ the HOMFLYPT polynomial to identify which specific segments in an open or closed chain support a knot and to specify 
the knot type by systematically checking all subsegments of increasing size until knotted segments are identified. By analyzing larger segments containing the knotted segment, we can also determine if it is a slipknot, i.e. is it contained in a larger unknotted segment. This provides a method by which we may determine the average lengths of knots and slipknots of specific types that occur. This allows us to determine the extent to which knotting is a local or a global matter. We will say that the average knotting is global if the average length of knots is proportional to length of the chain, $A n$, for large $\mathrm{n}$. The average knotting will be said to be weakly local if its extent is bounded by $A n^{\sigma}$, with $\sigma<1$, for large n. And, finally, we will say that the average knotting is strongly local if its extent is bounded by a constant $A$.

Conjecture 5.1. On average, knotting and slipknotting is strongly local in random walks.

Recent simulation data, see Figure 20, suggests that this conjecture is true with the average length of knots bounded by 25 and the average length of slipknots bounded by 50 . This is likely to be a challenging theoretical problem as we know that both local and global knots occur with probability one as the length of the walk goes to infinity. ${ }^{10,13,48,49}$

For random polygons in the simple cubic lattice, there is strong evidence that the knot size scales as the $\frac{3}{4}$ power ${ }^{15,18,50}$ and is, therefore, weakly local.

Problem 5.6. How do knots and slipknots in equilateral 3-space polygons scale?

\subsubsection{Implications of knot and slipknot localization on the average shape}

If knot localization in equilateral polygons is sufficiently strong, the characteristics of the random unknotted polygon would determine the average asymptotic spatial properties for any fixed knot type. On the other hand, when one considers the entire populaltion of $n$-gons, the contributions from the exponential growth in numbers of increasingly complex compact knots appears to have a measurable influence on the average properties. For example, this accounts for the existence of equilibrium lengths of polygons having fixed knot types. ${ }^{51-54}$ These powerful phenemona appear to account for the principle influences of knotting on the polygonal models of macromolecules. 


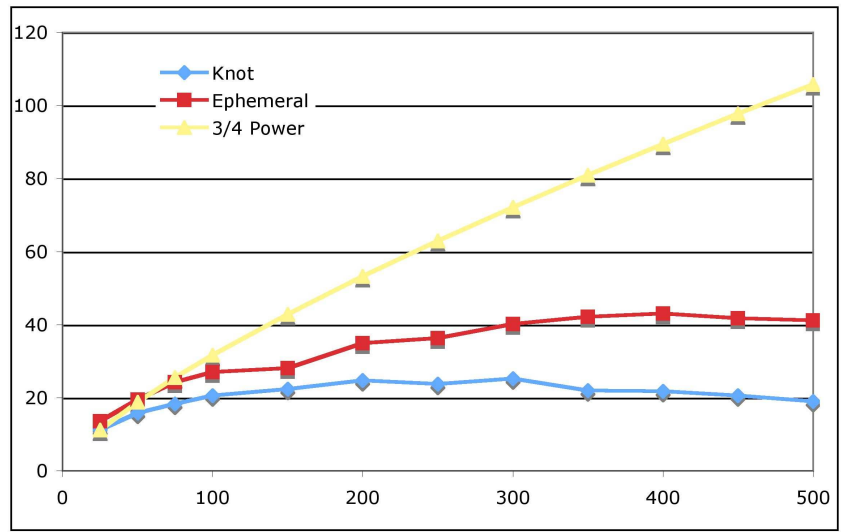

Fig. 20. Average knot localization in random walks

Conjecture 5.2. As the length of a walk or polygon of a fixed knot type increases, the average spatial properties converge to those of the unknot.

\subsubsection{Influence of knotting on physical properties: squared radius of gyration}

The squared radius of gyration, the average squared distance from the center of mass, is the principal physical measure of the size of a polymer. In the case of polygonal models, the mass is concentrated at the vertices and the squared radius of gyration is the average of the squared distance of the vertices from the center of mass. Polymers at thermodynamic equilibrium are modeled by random walks of two types. In good solvents, they are modeled by self-avoiding random walks as segments do not approach each other due to the repulsive forces between non-adjacent segments. Polymers chains under the $\theta$ condition (where segments neither repel nor attract) and for polymers in the dense melt phase (where individual chains are entangled with many others) are modeled by ideal random walks. For non-selfavoiding random walks, e.g. those in 3 -space, $\left\langle R_{G}{ }^{2}\right\rangle$, is proportional to $N^{2 \nu}$, where $N$ is the number of steps or segments, with $\nu \approx 0.500$. For selfavoiding random walks, $\left\langle R_{G}{ }^{2}\right\rangle$, is proportional to $N^{2 \nu}$, with $\nu \approx 0.588$. A. Grosberg ${ }^{55}$ argued that, in the long chain regime, polymers with effective diameter zero of a fixed knot type should have scaling exponent $\nu$ of 0.588 and that the length required for this behavior should increase with the complexity of the knot. Dobay et. al. ${ }^{51}$ showed that random walks, without 
excluded volume, have radius of gyration that scales at 0.500 but individual knot types (including the unknot) have radius of gyration scaling at 0.588 .

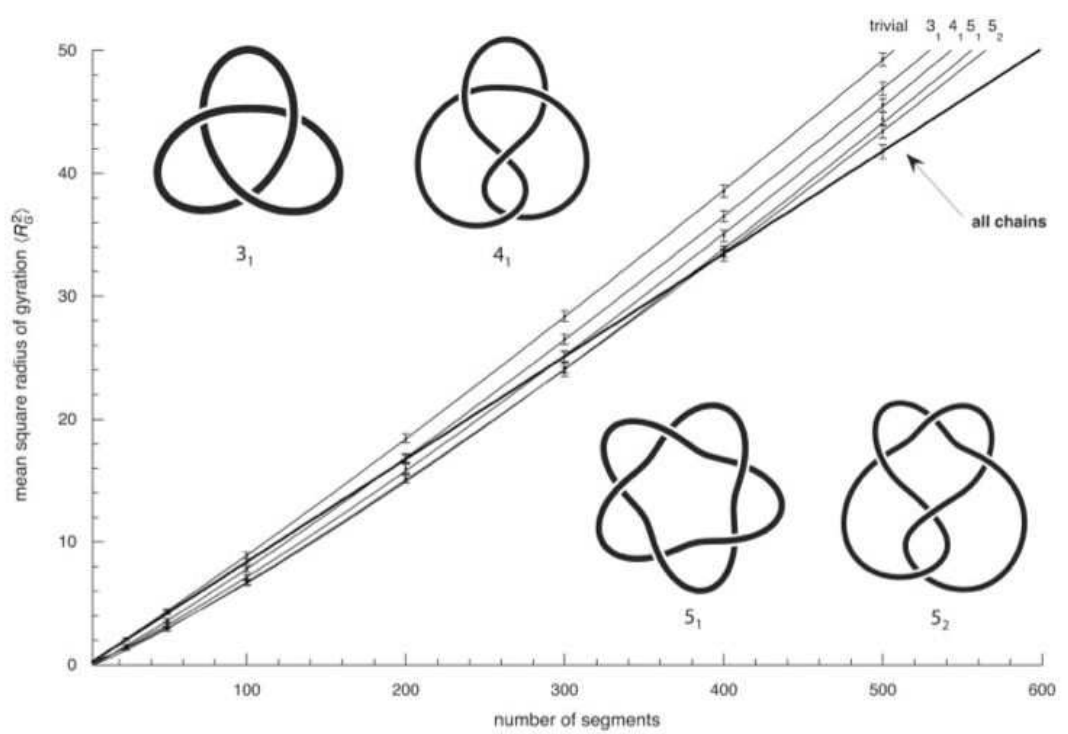

Fig. 21. Closed polygons: average squared radius of gyration for all types and specified knot types showing the difference in scaling. ${ }^{51}$

The equilibrium length is the number of edges at which the average property, e.g. the radius of gyration, equals the average of the entire population. In Figure 21, the crossing behaviour that defines the radius of gyration equilibrium lengths for the simplest knot types are shown.

One can make the same analysis of for random walks in which the knot types are identified using the MDS method. ${ }^{7}$ The scaling of the squared radius of gyration is determined for knotted random walks and the associated equilibrium length is defined, see Figure 22.

\subsubsection{Influence of knotting on physical properties: average crossing number}

The average crossing number, ACN, is one of the most frequently used measures of entanglement, an intuitive but informal concept that plays a fundamental role in understanding the physical properties of many systems. For random polygons, Diao et $\mathrm{al}^{51}$ show the scaling of the ACN is 


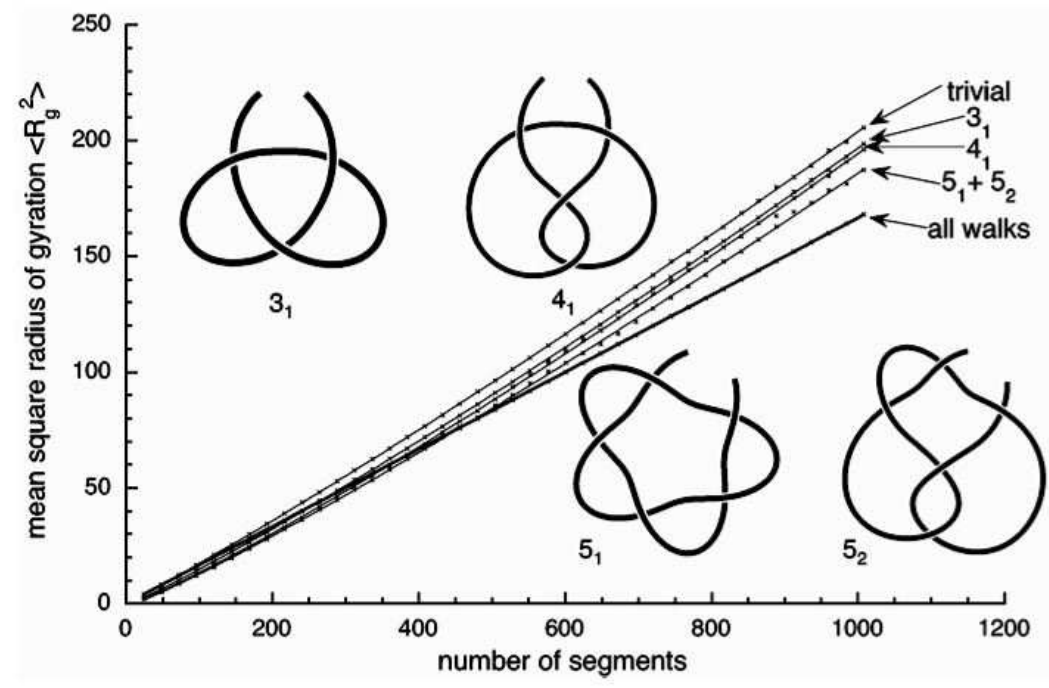

Fig. 22. Open polygons: average squared radius of gyration for all types and specified knot types in random walks, showing the difference in scaling. ${ }^{7}$

$\frac{3}{16} n \ln (n)+O(n)$. Here, again, the increasing presence of more and more complex knots causes the existence of equilibrium lengths for individual knot types, see Figure 23.

\subsubsection{Influence of knotting on geometric properties: total curvature and total torsion}

The Fary-Milnor Theorem 3.1 states that the total curvature of a polygonal knot is bounded below by $2 \pi B r(K)$, where $B r(K)$ is the bridge number of the knot type, a measure of complexity of the knot type. When one considers the family of equilateral polygons realizing the knot type, what is the average total curvature and, for that matter, the average total torsion? These give geometric measures of the complexity of the knotted polymer models. ${ }^{56}$ To better understand the geometry and topology of ring polymer chains under $\theta$ conditions or in the melt phase, we consider the statistics of the total curvature and total torsion as the number of edges increases. A little reflection leads to the realization that the dominant term is $\frac{\pi}{2} n$, where $n$ is the number of edges. Subtracting this dominant term allows one to analyze the second order terms, shown in Figure 24. There is visibly sufficient sensitivity to the presence of knotting to show again the existence 


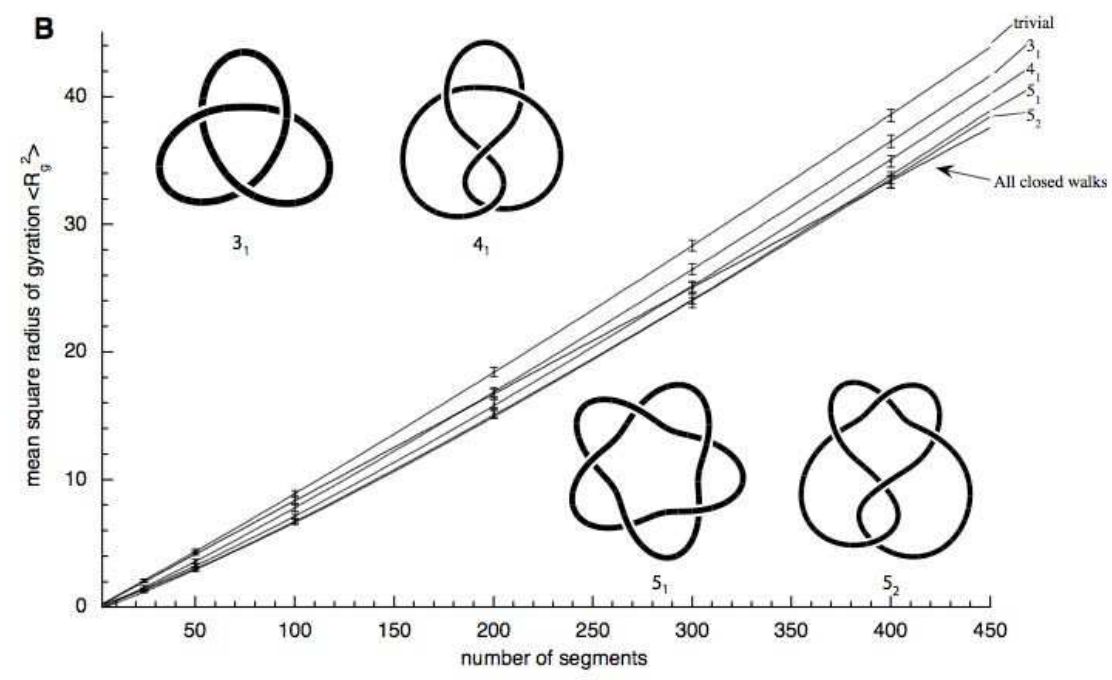

Fig. 23. The ACN and associated equilibrium lengths ${ }^{51}$

of equilibrium lengths for the total curvature.

We noted that the average second order effect for total curvature and total torsion over all configurations appeared to be constant, only differing in sign. Indeed, this is the case as was proved by Grosberg ${ }^{57}$ who showed that this value was exactly $\frac{3 \pi}{8}$. Specifically, he showed that the average total curvature of phantom polygons is asymptotically equal to $\frac{n \pi}{2}+\frac{3 \pi}{8}+O\left(\frac{1}{n}\right)$. If we let et $\left\{e_{i}\right\}_{i=1, \ldots, n}$ denote the set of edge vectors of a random polygon, then $\sum e_{i}=0$. Taking the average of the inner product give $\left\langle e_{i} \cdot e_{j}\right\rangle=\frac{-1}{n-1}$ which leads to $\langle c u r v\rangle=\frac{n \pi}{2}+\frac{3 \pi}{8}$.

Figure 25 shows a comparision of these equilibrium lengths to experimentally observed gel separation of knotted DNA, a traditional means to compare the degree to which these quantities might correlate to physically observed behavior.

Problem 5.7. How does the shape of macromolecules depend upon knotting?

\section{Concluding remarks}

In this report, I have described just a few of the more interesting features of the mathematical theory of knotting and linking that are deeply connected 

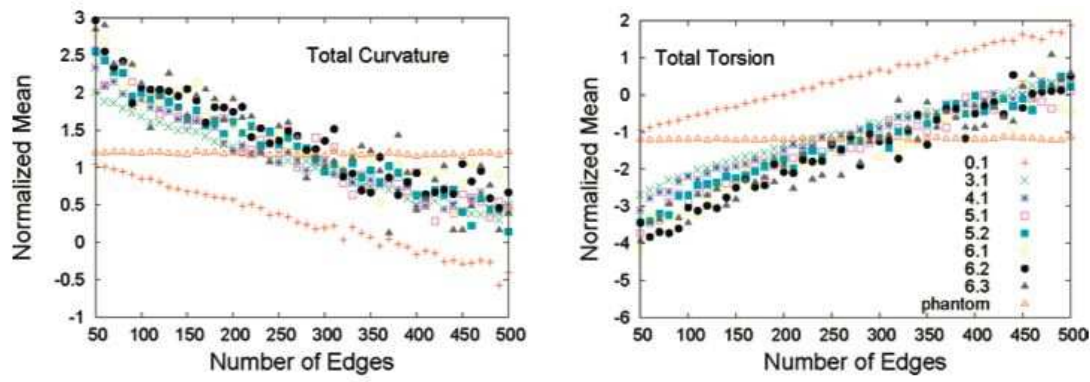

Fig. 24. The normalized total torsion and total curvature of random poygons. ${ }^{56}$

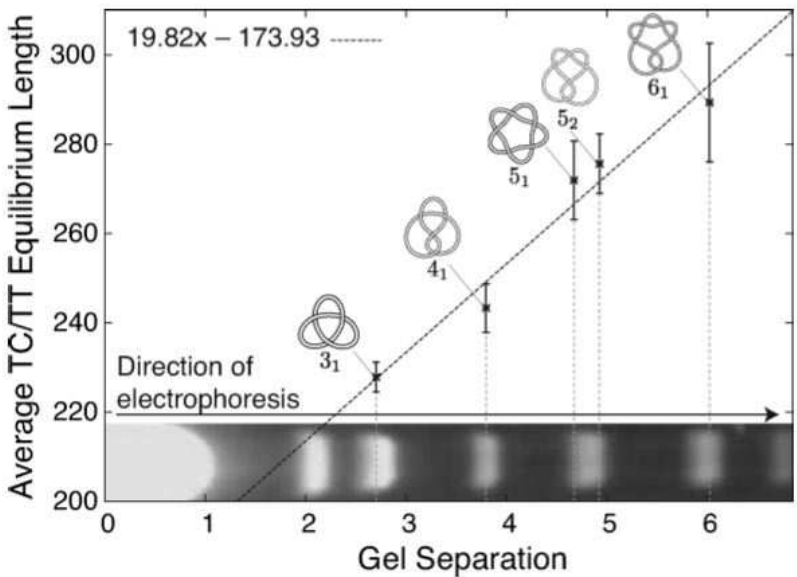

Fig. 25. Total curvature equilibrium lengths versus gel separation. ${ }^{56}$

with natural phenomena. Some of these are truly geometric in nature, others are topological, and many of the concepts and problems arise due to the interest in biological and physical polymers, especially the structure of DNA and proteins. As rigorous mathematical analysis is often beyond present reach, much of the effort to forge and understand these relationships and their implications has been numerical in nature whereby one wishes to randomly sample the population of configurations associated to an appropriate mathematical model. As a consequence, we have employed two key procedures, the crankshaft rotations and the polygonal folds, each providing a method taking any equilateral polygon in 3-space to any other in a 
finite sequence of steps. These procedures can then be used to randomly sample the space of polygons and generate sets of data which are then used to illuminate a number of these questions. Finally, we have looked at several applications to questions concerning the influence of knotting on the spatial structure of macromolecules. In the course of discussing these results, we have cited outstanding problems and conjectures. Some are quite accesible while others, I believe, are very challenging and may well require the development of new mathematical methods.

I hope these lecture notes have provided an introduction to a few of the more interesting facets of physical knot theory and their connections with a topological and geometric knot theory, as well as some newer research areas which one might better describe as statistical and computational knot theory. The interactions with the students and my fellow particiants in the ICTP Advanced School and Conference on Knot Theory and its Applications to Physics and Biology was a truly memorable experience. I wish to express my appreciation to Le Dung Trang and his staff and especially to the organizers, Slavik Jablan, Louis Kauffman, Sofia Lambropoulou and Jozef Przytycki for their outstanding leadership in organizing this opportunity to share our passion for research with the most diverse group of students and researchers that I have even encountered.

\section{References}

1. J. Cantarella and H. Johnston, J. Knot Theory Ramifications 7, 1027 (1998).

2. T. Biedl, E. Demaine, M. Demaine and et al., Discrete Comput. Geom. 26, 269 (2001).

3. G. Toussaint, Beiträge Algebra Geom. 42, 301 (2001).

4. E. D. Demaine, S. Langerman, J. O'Rourke and J. Snoeyink, Comput. Geom. 26, 37 (2003), Thirteenth Canadian Conference on Computational Geometry-CCCG'01 (Waterloo, ON).

5. G. Aloupis, G. Ewald and G. Toussaint, Beiträge Algebra Geom. 45, 429 (2004).

6. T. J. Clark and G. A. Venema, J. Knot Theory Ramifications 13, 479 (2004).

7. K. Millett, A. Dobay and A. Stasiak, Macromolecules 38, 601 (2005).

8. K. C. Millett and B. M. Sheldon, Tying down open knots: a statistical method for identifying open knots with applications to proteins, in Physical and numerical models in knot theory, , Ser. Knots Everything Vol. 36 (World Sci. Publ., Singapore, 2005), pp. 203-217.

9. K. C. Millett, J. Knot Theory Ramifications (2010).

10. D. W. Sumners and S. G. Whittington, J. Phys. A 21, 1689 (1988).

11. E. J. Janse van Rensburg, D. A. W. Sumners, E. Wasserman and S. G. Whittington, J. Phys. A 25, 6557 (1992).

12. M. Mansfield, Structural Biology 1, 213 (1994). 
13. Y. Diao, J. C. Nardo and Y. Sun, Journal of Knot Theory and its Ramifications 10, 597 (2001).

14. W. Taylor, Nature 406, 916 (2000).

15. B. Marcone, E. Orlandini, A. L. Stella and F. Zonta, J. Phys. A 38, L15 (2005).

16. P. Virnau, L. A. Mirny and M. Kardar, PLoS Computational Biology 2, p. e122(September 2006).

17. G. Kolesov, P. Virnau, M. Kardar and L. A. Mirny, Nucleic Acids Res. 35, w425 (2007).

18. B. Marcone, E. Orlandini, A. L. Stella and F. Zonta, Physical Review E (Statistical, Nonlinear, and Soft Matter Physics) 75, p. 041105 (2007).

19. M. Laso, N. C. Karayiannis, K. Foteinopoulou, M. L. Mansfield and M. Kroger, Soft Matter 5, 1762 (2009).

20. P. Freyd, D. Yetter, J. Hoste, W. B. R. Lickorish, K. Millett and A. Ocneanu, Bull. Amer. Math. Soc. (N.S.) 12, 239 (1985).

21. M. P. do Carmo, Differential geometry of curves and surfaces (Prentice-Hall Inc., Englewood Cliffs, N.J., 1976). Translated from the Portuguese.

22. R. S. Millman and G. D. Parker, Elements of differential geometry (PrenticeHall Inc., Englewood Cliffs, N. J., 1977).

23. J. W. Milnor, Ann. of Math. 52, 248 (1950).

24. O. Krötenheerdt and S. Veit, On the theory of solid knots, in Physical and Numerical Models in Knot Theory, eds. J. A. Calvo, K. C. Millett, E. J. Rawdon and A. Stasiak, Ser. Knots Everything, Vol. 36 (World Sci. Publishing, Singapore, 2005) pp. 1-18. Translated by Ted Ashton.

25. O. Gonzalez and J. H. Maddocks, Proc. Natl. Acad. Sci. USA 96, 4769 (1999).

26. E. J. Rawdon, The thickness of polygonal knots, $\mathrm{PhD}$ thesis, University of Iowa1997.

27. K. C. Millett and E. J. Rawdon, J. Comput. Phys. 186, 426 (2003).

28. J. Baranska, P. Pieranski and E. J. Rawdon, Ropelength of tight polygonal knots, in Physical and Numerical Models in Knot Theory, eds. J. A. Calvo, K. C. Millett, E. J. Rawdon and A. Stasiak, Ser. Knots Everything, Vol. 36 (World Sci. Publishing, Singapore, 2005) pp. 293-321.

29. R. Randell, A molecular conformation space, in Studies in Physical and Theoretical Chemistry, ed. R. Lacher, MATH/CHEM/COMP 1987, Vol. 54 (Elsevier Science, Amsterdam, 1988) pp. 125-140.

30. R. Randell, Conformation spaces of molecular rings, in Studies in Physical and Theoretical Chemistry, ed. R. Lacher, MATH/CHEM/COMP 1987, Vol. 54 (Elsevier Science, Amsterdam, 1988) pp. 141-156.

31. J. A. Calvo, Geometric knot theory: the classification of spatial polygons with a small number of edges, $\mathrm{PhD}$ thesis, University of California, Santa Barbara1998.

32. J. A. Calvo, J. Knot Theory Ramifications 10, 245 (2001), Knots in Hellas '98, Vol. 2 (Delphi).

33. J. A. Calvo, Characterizing polygons in $\mathbb{R}^{3}$, in Physical knots: knotting, linking, and folding geometric objects in $\mathbb{R}^{3}$ (Las Vegas, NV, 2001), , Contemp. Math. Vol. 304 (Amer. Math. Soc., Providence, RI, 2002) pp. 37-53. 
34. J. A. Calvo and K. C. Millett, Minimal edge piecewise linear knots, in Ideal knots, (World Sci. Publishing, Singapore, 1998) pp. 107-128.

35. J. A. Calvo, Characterizing polygons in $\mathbb{R}^{3}$, in Physical knots: knotting, linking, and folding geometric objects in BbbR (Las Vegas, NV, 2001), , Contemp. Math. Vol. 304 (Amer. Math. Soc., 2001).

36. S. Alvarado, J. Calvo and K. Millett, Journal of Statistical Physics 143, 102 (2011), 10.1007/s10955-011-0164-4.

37. K. C. Millett, J. Knot Theory Ramifications 3, 263 (1994), Random knotting and linking (Vancouver, BC, 1993).

38. M. Kapovich and J. Millson, J. Differential Geom. 44, 479 (1996).

39. G. Toussaint, Computational Geometry 31, 219 (2005).

40. R. Larsen and M. Marx, An Introduction to Mathematical Statistics and Its Applications, 3 edn. (Prentice Hall, Upper Saddle River, New Jersey, 2001).

41. B. Ewing and K. C. Millett, Computational algorithms and the complexity of link polynomials, in Progress in knot theory and related topics, (Hermann, Paris, 1997) pp. 51-68.

42. B. Ewing and K. C. Millett, A load balanced algorithm for the calculation of the homfly polynomial, in The Mathematical Heritage of C. F. Gauss, (World Scientific Publishing Co., 1991) pp. 225-266.

43. W. B. R. Lickorish and K. C. Millett, Topology 26, 107 (1987).

44. J. H. Przytycki and P. Traczyk, Proc. Amer. Math. Soc. 100, 744 (1987).

45. K. C. Millett and D. W. Sumners (eds.), Random knotting and linking (World Sci. Publishing, Singapore, 1994). Papers from the AMS Special Session held in Vancouver, BC, August 15-19, 1993.

46. K. C. Millett, Monte carlo explorations of polygonal knot spaces, in Proceedings of the International Conference on Knot Theory and its Ramifications held in Delphi, August 7-15, 1998, eds. C. M. Gordon, V. F. R. Jones, L. H. Kauffman, S. Lambropoulou and J. H. Przytycki (World Sci. Publishing, Singapore, 2001).

47. K. C. Millett and E. J. Rawdon, Universal characteristics of polygonal knot probabilities, in Physical and Numerical Models in Knot Theory, eds. J. A. Calvo, K. C. Millett, E. J. Rawdon and A. Stasiak, Ser. Knots Everything, Vol. 36 (World Sci. Publishing, Singapore, 2005) pp. 247-274.

48. N. Pippenger, Discrete Appl. Math. 25, 273 (1989).

49. Y. Diao, N. Pippenger and D. W. Sumners, J. Knot Theory Ramifications 3, 419 (1994), Random knotting and linking (Vancouver, BC, 1993).

50. E. Orlandini, A. L. Stella, C. Vanderzande and F. Zonta, J. Phys. A 41, 122002, 7 (2008).

51. Y. Diao, A. Dobay, R. B. Kusner, K. C. Millett and A. Stasiak, J. Phys. A 36, 11561 (2003).

52. A. Dobay, J. Dubochet, K. Millett, P.-E. Sottas and A. Stasiak, Proc. Natl. Acad. Sci. USA 100, 5611 (2003).

53. E. J. Rawdon, A. Dobay, J. C. Kern, K. C. Millett, M. Piatek, P. Plunkett and A. Stasiak, Macromolecules 41 (2008).

54. K. C. Millett, Plunkett, M. Patrick, Piatek, E. J. Rawdon and A. Stasiak, The Journal of Chemical Physics 130, 165104 (2009). 
55. A. Y. Grosberg, Phys. Rev. Lett. 85, 3858 (2000).

56. P. Plunkett, M. Piatek, A. Dobay, J. Kern, K. Millett, A. Stasiak and E. Rawdon, Macromolecules 40, 3860 (2007).

57. A. Y. Grosberg, Macromolecules 41, 4524 (2008). 